\title{
Expressions for the Entropy of Binomial-Type Distributions*
}

\author{
MAHDi CheRAGHChI ${ }^{\dagger}$ \\ Department of Computing \\ Imperial College London \\ London, UK
}

\begin{abstract}
We develop a general method for computing logarithmic and log-gamma expectations of distributions. As a result, we derive series expansions and integral representations of the entropy for several fundamental distributions, including the Poisson, binomial, beta-binomial, negative binomial, and hypergeometric distributions. Our results also establish connections between the entropy functions and to the Riemann zeta function and its generalizations.
\end{abstract}

\section{Introduction}

Deriving expressions for the Shannon entropy of commonly studied distributions is of fundamental significance to information and communication theory, statistics, and theoretical computer science. For many distributions, exact closed-form expression for the entropy is known. A noncomprehensive list of such distributions include uniform, Bernoulli, geometric, exponential, Laplace, normal, log-normal, Pareto, Cauchy, Weibull, Rayleigh, t-distribution, Dirichlet, Wishart, Chisquared, scaled inverse chi-squared, gamma, and, inverse-gamma distribution. In many cases, the entropy is a simple expression in terms of the first few moments, and in other cases, the logarithmic expectation of the distribution takes a tractable form. For many fundamental distributions, however, we do not expect to have direct, closed form, expressions for the entropy in terms of elementary, or common special, functions. In such cases, high quality approximations, series expansions, or integral forms, for the entropy is desirable.

In this work, we focus on what we call "binomial-type" distributions, that exhibit factorial terms in their expressions for the probability mass function. In fact, we derive a general expression for computing log-gamma expectations; i.e., expressions of the form $\mathbb{E}[\log \Gamma(\alpha+X)]$ for any distribution in terms of its moment generating function. Specific examples that we will use to demonstrate our technique includes Poisson, binomial, beta-binomial, negative binomial, and hypergeometric distributions. We recall that the binomial distribution is defined to capture the number of success events in a series of $n$ Bernoulli trials, for a given parameter $n$ and a given success probability. This contains the Poisson distribution as a limiting case, and is in turn a limiting case for the more general beta-binomial distribution. A negative binomial distribution is defined similar to the binomial distribution, but with a varying number of trials and fixed number $r$ of success events. Finally, a hypergeometric distribution is regarded as an analogue of the binomial distribution where the Bernoulli trials are performed without replacement. Apart from their fundamental

* A preliminary version of this work appears in Proceedings of the 2018 IEEE International Symposium on Information Theory (ISIT).

${ }^{\dagger}$ Email: 〈m.cheraghchi@imperial.ac.uk〉. 
nature, the entropy of such distributions are of particular significance to information theory, for example in quantifying the number of bits required for compression of the corresponding sources. Moreover, understanding the entropy of binomial and Poisson distributions is a key step towards a characterization of the capacity of basic channels with synchronization errors, including the deletion and Poisson-repeat channels (cf. [Mit08]). Poisson entropy is also of key significance to the theory of optical communication and in understanding the capacity of Poisson-type channels in this context (cf. [Sha90, Ver99, Ver08, AW12]). Indeed, an integral expression for the log-gamma expectation of Poisson random variables (similar to one of our results) was the key to [Mar07, CR18] for obtaining sharp elementary estimates on the capacity of the discrete-time Poisson channel. On the other hand, similar integral representations, of the type we derive, were used in recent works of the author [Che18, CR18b] to derive strong upper bounds on the capacity of the binary deletion, Poisson-repeat, and related channels with synchronization errors.

For a wide range of the parameters, binomial-type distributions are approximated by a normal distribution via the central limit theorem. When the variance of these distributions is large (and, for the negative binomial distribution, the order parameter $r$ is large), the entropy is quite accurately estimated by the entropy of a normal distribution with matching variance. However, as the variance decreases, the quality of this approximation deteriorates. In fact, as the variance $\sigma$ tends to zero, the entropy of a normal distribution, which is $\frac{1}{2} \log \left(2 \pi e \sigma^{2}\right)$, diverges to $-\infty$, while the actual entropy of the underlying distribution tends to zero. In such cases, a more refined expression for the entropy in terms of a convergent power series or integral expression would correctly capture its behavior.

Our starting point is a simple, but curious, manipulation of the entropy expression for the Poisson distribution. Recall that a Poisson distribution is defined by the probability mass function

$$
\operatorname{Poi}(k ; \lambda)=\frac{\lambda^{k} e^{-\lambda}}{k !}
$$

where $k \in\{0,1, \ldots\}$, and the parameter $\lambda>0$ is the mean and variance of the distribution. The entropy of this distribution is thus equal, by the definition of Shannon entropy, to

$$
H_{\text {Poi }}(\lambda):=-\sum_{k=0}^{\infty} \operatorname{Poi}(k ; \lambda) \log (\operatorname{Poi}(k ; \lambda))=-\lambda \log (\lambda / e)+e^{-\lambda} \sum_{k=0}^{\infty} \frac{\lambda^{k} \log k !}{k !} .
$$

As mentioned above, $H_{\mathrm{Poi}}(\lambda)$ tends to $\frac{1}{2} \log \left(2 \pi e \sigma^{2}\right)$ as $\lambda$ grows. A more accurate estimate is given in [EB88]; namely, that for large $\lambda$,

$$
H_{\mathrm{Poi}}(\lambda)=\frac{1}{2} \log (2 \pi e \lambda)-\frac{1}{12 \lambda}-\frac{1}{24 \lambda^{2}}-\frac{19}{360 \lambda^{3}}+O\left(\lambda^{-4}\right) .
$$

We remark that asymptotic expansions for the entropy of binomial and negative binomial distributions for large mean (in terms of the difference between the entropy and the Gaussian entropy estimate) has been derived in [JS99] and [CGKK13] (and, among other results, in [DV98] and [Kne98]).

Let $X$ be a Poisson distributed random variable with mean $\lambda$, so that the second term on the 
right hand side of (1) becomes $E_{\mathrm{Poi}}(\lambda):=\mathbb{E}[\log X !]$. We now write, using the convolution formula,

$$
\begin{aligned}
E_{\text {Poi }}(\lambda) & =e^{-\lambda} \sum_{k=0}^{\infty} \frac{\lambda^{k} \log k !}{k !} \\
& =\sum_{j=0}^{\infty} \lambda^{j} \sum_{k=0}^{j} \frac{(-1)^{j-k}}{(j-k) !} \frac{\log k !}{k !} \\
& =\sum_{j=0}^{\infty} \frac{\lambda^{j}}{j !} \sum_{k=0}^{j}\left(\begin{array}{l}
j \\
k
\end{array}\right)(-1)^{j-k} \log k ! \\
& =\sum_{j=0}^{\infty} \frac{(-\lambda)^{j}}{j !} c(j),
\end{aligned}
$$

where we have defined the coefficients $c(j):=\sum_{k=0}^{j}\left(\begin{array}{l}j \\ k\end{array}\right)(-1)^{k} \log k$ !. The first few values for the $c(j), j=0,1, \ldots$, are (see integer sequences A122214 and A122215)

$$
\begin{gathered}
\log \left\{1,1,2, \frac{4}{3}, \frac{32}{27}, \frac{4096}{3645}, \frac{67108864}{61509375}, \frac{4503599627370496}{4204742431640625}, \frac{2535301200456458802993406410752}{2396825584582984447479248046875}\right\} \\
\approx\{0,0,0.693147,0.287682,0.169899,0.116655,0.0871265,0.068664,0.0561673\}
\end{gathered}
$$

where the logarithms are taken to base $e$. We have thus derived the Maclaurin series expansion of the function $E_{\mathrm{Poi}}(\lambda)$, which converges for all $\lambda>0$. If we formally replace each coefficient $c(j)$ in (2) with $\left(e^{t}-1\right)^{j}$, the expression turns into the power series expansion of $e^{\lambda\left(e^{t}-1\right)}$, which is the moment generating function of the Poisson distribution. Our main observation is that this is not a coincidence, and in fact the same phenomenon occurs for the log-gamma expectation of any (discrete or continuous) distribution defined over the non-negative reals. In particular, we prove the following:

Theorem 1. Let $M(t)$ be the moment generating function of any (continuous or discrete) distribution with mean $\mu=M^{\prime}(0)$, and $\alpha>0$ be a parameter. Suppose $M(t)$ is analytic around $t=0$ and let $Q(z):=M(\log (z+1))$ be represented by power series $Q(z)=1+\sum_{j=1}^{\infty} q(j) z^{j}$. Then, for $a$ random variable $X$ sampled from the distribution given by $M$, we have the following:

$$
\begin{aligned}
\mathbb{E}[\log \Gamma(X+\alpha)] & =\log \Gamma(\alpha)+\sum_{j=1}^{\infty}(-1)^{j} q(j) c_{\alpha}(j) \\
& =\log \Gamma(\alpha)+\int_{0}^{\infty}\left(\frac{\mu e^{-t}}{t}-\frac{e^{-\alpha t}(1-M(-t))}{t\left(1-e^{-t}\right)}\right) d t \\
& =\log \Gamma(\alpha)+\mu \log \alpha-\int_{0}^{1} \frac{(1-z)^{\alpha-1}}{z \log (1-z)}(Q(-z)+\mu z-1) d z \\
\mathbb{E}[\log (X+\alpha)] & =\sum_{j=1}^{\infty}(-1)^{j} q(j-1) c_{\alpha}(j) \\
& =\int_{0}^{\infty} \frac{e^{-t}-e^{-\alpha t} M(-t)}{t} d t
\end{aligned}
$$

where

$$
c_{\alpha}(j):=-\sum_{k=0}^{j-1}(-1)^{k}\left(\begin{array}{c}
j-1 \\
k
\end{array}\right) \log (k+\alpha) .
$$


Proof of the above theorem turns out to be remarkably simple, yet it provides a general and powerful tool for deriving series expansions and integral expressions for the entropy of distributions involving factorial terms in their probability mass functions, a task that may seem elusive by a direct approach.

\subsection{Summary of the main results}

We apply Theorem 1 to derive series and integral expressions for the entropy and log-gamma expectations of several distributions over the non-negative integers. We will particularly consider the Poisson, binomial, beta-binomial, negative binomial, and hypergeometric distributions that are respectively defined by the probability mass functions below ${ }^{1}$ (for $k=0,1, \ldots$ ):

$$
\begin{array}{rlrl}
\operatorname{Poi}(k ; \lambda) & =\frac{\lambda^{k} e^{-\lambda}}{k !}, & \lambda>0 \\
\operatorname{Bin}(k ; n, p) & =\left(\begin{array}{c}
n \\
k
\end{array}\right) p^{k}(1-p)^{n-k}, & n>0, p \in(0,1) \\
\operatorname{BBin}(k ; n, \alpha, \beta) & =\left(\begin{array}{c}
n \\
k
\end{array}\right) \frac{B(k+\alpha, n-k+\beta)}{B(\alpha, \beta)}, \alpha, \beta>0 \\
\operatorname{NBin}(k ; r, p) & =\left(\begin{array}{c}
k+r-1 \\
k
\end{array}\right) p^{k}(1-p)^{r}, & r>0, p \in(0,1) \\
\operatorname{HG}(k ; N, K, n) & =\frac{\left(\begin{array}{c}
K \\
k
\end{array}\right)\left(\begin{array}{c}
N-K \\
n-k
\end{array}\right)}{\left(\begin{array}{c}
N \\
n
\end{array}\right)}, & N, K, n>0
\end{array}
$$

where $B(\alpha, \beta)=\Gamma(\alpha) \Gamma(\beta) / \Gamma(\alpha+\beta)$ denotes the beta function. We remark that a similar technique has been employed in [Kne98] (and rediscovered in [Mar07]) to derive integral expressions for the entropy of Poisson, binomial, and negative binomial distributions.

Let $X_{\mathrm{Poi}}, X_{\mathrm{Bin}}, X_{\mathrm{BBin}}, X_{\mathrm{Bin}}$, and $X_{\mathrm{HG}}$ be random variables drawn from their respective distributions above. In Sections 4, 5, 6, 7, and 8, we derive the expressions below. All expressions converge for the whole range of parameters, and logarithms are taken to the base in which the entropy is measured.

$$
\begin{aligned}
H\left(X_{\text {Poi }}\right) & =-\lambda \log (\lambda / e)+\sum_{j=2}^{\infty} \frac{c(j)}{j !}(-\lambda)^{j} \\
& =-\lambda \log (\lambda / e)+\int_{0}^{1} \frac{1-e^{-\lambda z}-\lambda z}{z \log (1-z)} d z,
\end{aligned}
$$

where we use the convention ${ }^{2} c(j):=c_{1}(j)$.

$$
\begin{aligned}
H\left(X_{\mathrm{Bin}}\right) & =n h(p)+\int_{0}^{\infty}\left(\frac{\left(1-p+p e^{-t}\right)^{n}+\left(p+(1-p) e^{-t}\right)^{n}-e^{-n t}-1}{t\left(e^{t}-1\right)}\right) d t \\
& =n h(p)+\sum_{j=2}^{\infty}\left(\begin{array}{l}
n \\
j
\end{array}\right)(-1)^{j} c(j)\left(p^{j}+(1-p)^{j}-1\right)
\end{aligned}
$$

\footnotetext{
${ }^{1}$ We refer the reader to standard textbooks on probability (such as [DS12]) and information theory (such as [CT06]) for the standard definitions of the various notions used.

${ }^{2}$ We note that the expression for $c(j)$ in $(2)$ indeed coincides with $c_{1}(j)$ due to the fact that the logarithm function is the first finite derivative of log-gamma.
} 
where $h(p):=-p \log p-(1-p) \log (1-p)$ is the binary entropy function.

$$
H\left(X_{\mathrm{BBin}}\right)=-\log (n B(n, \alpha+\beta))+\sum_{j=2}^{\infty}\left(\begin{array}{l}
n \\
j
\end{array}\right) \frac{(-1)^{j}}{(\alpha+\beta)^{(j)}}\left(\left(c(j)-c_{\alpha}(j)\right) \alpha^{(j)}+\left(c(j)-c_{\beta}(j)\right) \beta^{(j)}\right),
$$

where we use the notation $a^{(j)}$ for the rising factorial $a(a+1) \cdots(a+j-1)$.

$$
\begin{aligned}
& \mathbb{E}\left[\log \Gamma\left(X_{\mathrm{BBin}}+r\right)\right]=\log \Gamma(r)+\frac{n \alpha \log r}{\alpha+\beta} \\
& +\int_{0}^{1} \frac{(1-z)^{r-1}\left({ }_{2} F_{1}(-n, \alpha ; \alpha+\beta ;-z)+n \alpha /(\alpha+\beta)-1\right)}{z \log (1-z)} d z \\
& =\log \Gamma(r)+\sum_{j=2}^{\infty}(-1)^{j} c_{r}(j) \frac{\alpha^{(j)}}{(\alpha+\beta)^{(j)}}\left(\begin{array}{c}
n \\
j
\end{array}\right) . \\
& H\left(X_{\mathrm{NBin}}\right)=\frac{r h(p)}{1-p}+\sum_{j=1}^{\infty}\left(\begin{array}{c}
j+r-1 \\
j
\end{array}\right)\left(\frac{p}{p-1}\right)^{j}\left(c(j)-c_{r}(j)\right) \\
& =\frac{r(h(p)-p \log r)}{1-p}+\int_{0}^{1} \frac{\left((1-z)^{r-1}-1\right)\left((1+p z /(1-p))^{-r}+p r z /(1-p)-1\right)}{z \log (1-z)} d z . \\
& H\left(X_{\mathrm{HG}}\right)=\log \left(\begin{array}{c}
N \\
n
\end{array}\right)-\log K !-\log (N-K) ! \\
& +\sum_{j=2}^{\infty} \frac{(-1)^{j} c(j)}{(N)_{j}}\left((K)_{j}(n)_{j}+(N-K)_{j}(n)_{j}+(K)_{j}(N-n)_{j}+(N-K)_{j}(N-n)_{j}\right) .
\end{aligned}
$$

We demonstrate examples of connections between the entropy functions via functional transformations as well as connections between them and the Riemann zeta function and its many generalizations. We believe that such connections will stimulate further research towards a full understanding of the entropy of such fundamental distributions as the Poisson, binomial, and related distributions.

Among our results, we show (in Section 4, Theorem 2) that the Laplace transform of $H\left(X_{\mathrm{Poi}}\right)$, regarded as a function of the expectation $\lambda$, is equal to

$$
\frac{\gamma+\log z}{z^{2}}-\frac{1}{z(1+z)} \Phi^{\prime}\left(\frac{1}{1+z}\right)
$$

where $\Phi^{\prime}$ is the derivative of the polylogarithm function as defined in (31). Another example is a connection between the geometric distribution (on $0,1, \ldots$ ) and the Poisson distribution. Letting $X_{\text {Geom }}$ denote a geometrically distributed random variable, we show (in (45)) that the logarithmic expectation $\mathbb{E}\left[\log \left(X_{\mathrm{Geom}}+1\right)\right]$, as a function of the mean of $X_{\mathrm{Geom}}$, generates the logarithmic difference coefficients $c_{\alpha}(j)$ defined in (8). Moreover, we express this function in terms of the Laplace transform of the entropy of a Poisson distribution (Section 7, Theorem 6). Finally, we derive connections between the $c_{\alpha}(j)$ and their generating function to the Riemann zeta function (Appendix A) and its generalizations (Section 2, (33)) that we believe may serve as a natural continuation point towards a complete understanding of the entropy of Poisson and related distributions. 


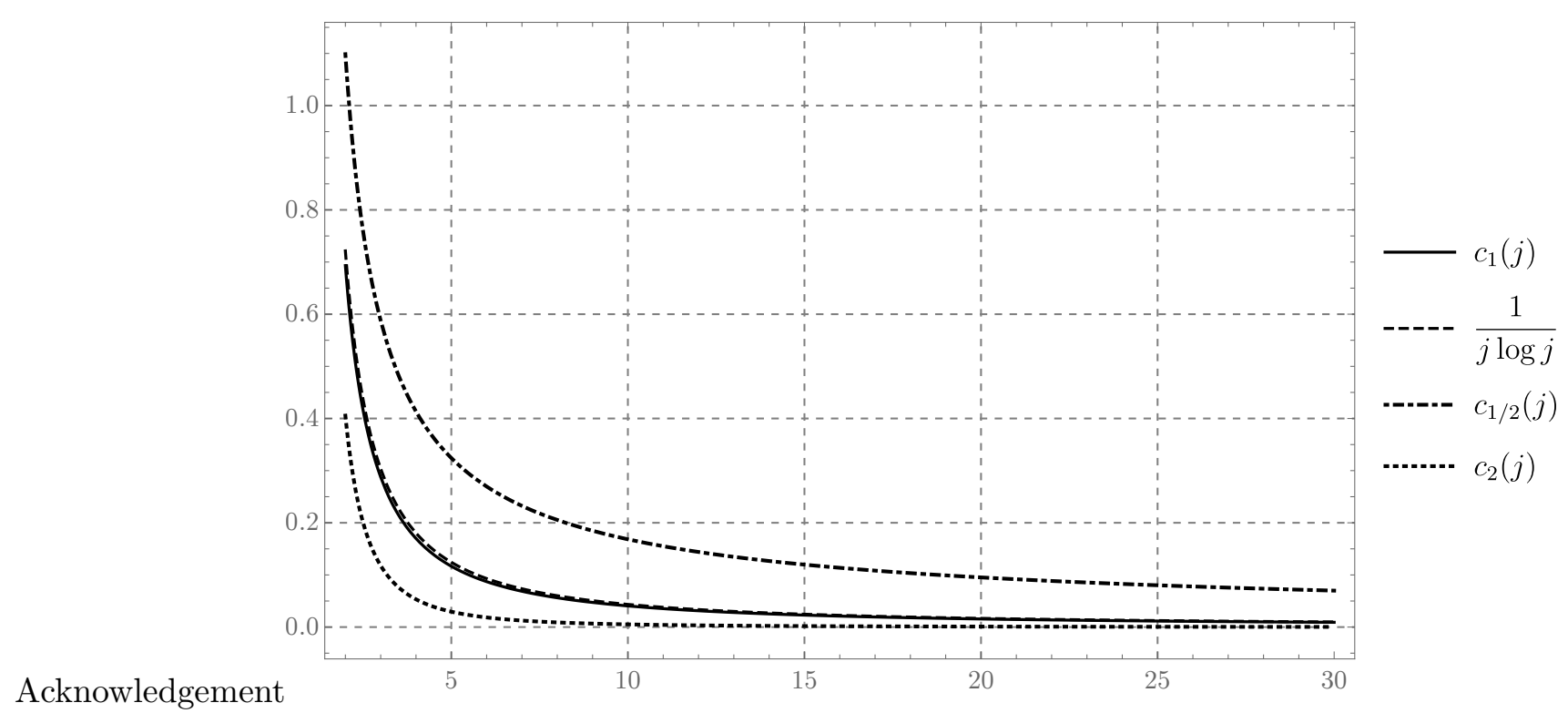

Figure 1: Logarithmic difference coefficients $c_{\alpha}(j)$ as a function of $j$.

Organization. The rest of the article is organized as follows. In Section 2, we study the coefficients $c_{\alpha}(j)$ in $(8)$ and their various properties. Section 3 gives a proof of Theorem 1 . In Sections 4, 5, 6, 7, and 8, we respectively apply Theorem 1 to obtain expressions for the entropy and log-gamma expectations of the Poisson, binomial, beta-binomial, negative binomial, and hypergeometric distributions. We conclude in Section 9 by a brief discussion of possible future directions and questions raised by this work.

\section{The logarithmic difference coefficients $c_{\alpha}(j)$}

The coefficients $c_{\alpha}(j)$ defined in (8) naturally appear in the analytic study of zeta functions (we will study an example related to this work in Appendix A) and are of fundamental importance in calculus of finite differences. They are essentially the Newton series expansion coefficients of the logarithm function around point $\alpha$, which is why we call them the logarithmic difference coefficients. The coefficients are plotted ${ }^{3}$ for various choices of $\alpha$ in Figure 1 . We observe that the function $1 /(j \log j)$ closely approximates $c_{1}(j)$.

Recall that the $j$ th forward difference of a function $f$ at point $\alpha$ is defined as

$$
\Delta^{j}[f](\alpha):=\sum_{k=0}^{j}\left(\begin{array}{l}
j \\
k
\end{array}\right)(-1)^{j-k} f(k+\alpha),
$$

or equivalently, as the $j$-fold application of the discrete derivative $\Delta[f](\alpha):=f(\alpha+1)-f(\alpha)$ of the function at $\alpha$ (and letting $\Delta^{0}[f](\alpha)=f(\alpha)$. Furthermore, we recall that the Newton series expansion of a function $f$ around point $\alpha$ is given by

$$
f(x+\alpha)=\sum_{j=0}^{\infty}\left(\begin{array}{l}
x \\
j
\end{array}\right) \Delta^{j}[f](\alpha),
$$

\footnotetext{
${ }^{3}$ The plot depicts the continuous interpolation of the $c_{\alpha}(j)$ given by $(27)$, so that the values are meaningful for non-integer choices of $j$ as well.
} 
which is the same as the formula for the Taylor expansion with continuous derivatives replaced by forward differences and powers of $x$ replaced by factorial powers. Note that $x$ need not be an integer. Applying (25) on $f(x)=\log x$, and comparing with (8), we see that

$$
\Delta^{j}[\log ](\alpha)=(-1)^{j+1} c_{\alpha}(j+1),
$$

so that

$$
\log (x+\alpha)=\sum_{j=0}^{\infty}\left(\begin{array}{l}
x \\
j
\end{array}\right)(-1)^{j+1} c_{\alpha}(j+1) .
$$

Unlike the Taylor expansion, one can verify that the above series converges to $\log (x+\alpha)$ for all values of $x>-\alpha$.

The coefficients $c_{\alpha}(j)$ have a compact integral representation. When $j>1$, we have

$$
c_{\alpha}(j)=\int_{0}^{\infty} \frac{\left(1-e^{-t}\right)^{j-1} e^{-\alpha t}}{t} d t
$$

which can be readily verified by a binomial expansion of the integrand and using the following basic identity (which holds for any $n>0$ ) on each resulting term:

$$
\int_{0}^{\infty} \frac{e^{-t}-e^{-n t}}{t} d t=\log n
$$

It is worthwhile to understand the generating function for the coefficients $c_{\alpha}(j)$. In order to do so, we start by recalling Lerch transcendent (cf. [EMOT53, p. 27])

$$
\Phi(z, s, \alpha):=\sum_{k=0}^{\infty} \frac{z^{k}}{(k+\alpha)^{s}}=\frac{1}{\Gamma(s)} \int_{0}^{\infty} \frac{t^{s-1} e^{-\alpha t}}{1-z e^{-t}} d t .
$$

By taking derivative of the above series with respect to $s$ at $s=0$, we obtain the generating function of the logarithmic sequence

$$
\Phi_{\alpha}^{\prime}(z):=\left.\frac{d}{d s} \Phi(z, s, \alpha)\right|_{s=0}=-\sum_{k=0}^{\infty} \log (k+\alpha) z^{k} .
$$

When $\alpha=1$, in which case we drop the subscript from the notation, the above can be written in terms of the polylogarithm function $\operatorname{Li}_{s}(z):=\sum_{k=1}^{\infty} z^{k} k^{-s}$ as

$$
\Phi^{\prime}(z):=\Phi_{1}^{\prime}(z)=\frac{d}{d s} \operatorname{Li}_{s}(z) /\left.z\right|_{s=0}
$$

Now, observe that the coefficient sequence $\left(-c_{\alpha}(j+1)\right)_{j=0}^{\infty}$ is the binomial transform $[\mathrm{Knu} 73$, p. 136] of the sequence $(\log (k+\alpha))_{k=0}^{\infty}$. Let

$$
C_{\alpha}(z):=\sum_{j=0}^{\infty} c_{\alpha}(j) z^{j}
$$

be the generating function for $\left(c_{\alpha}(j)\right)_{j=0}^{\infty}$, where we define $c_{\alpha}(0):=0$. Although we may treat the series formally, note that (32) converges when $|z|<1$ or $z=-1$ (since $c_{\alpha}(j)$ is a decreasing 
sequence for $\alpha>0$ ). Using (29) and the formula for generating function of the binomial transform, we thus have

$$
C_{\alpha}(z)=\frac{z}{1-z} \Phi_{\alpha}^{\prime}\left(\frac{-z}{1-z}\right)
$$

In this regard, (3) in Theorem 1 can be interpreted as the assertion that the log-gamma expectation $\mathbb{E}[\log \Gamma(X+\alpha)]-\log \Gamma(\alpha)$ of any distribution over non-negative reals is the inner product of the power series coefficients of $C_{\alpha}(-z)$ and that of the function $Q(z):=M(\log (z+1))$ derived from the moment generating function (the power series coefficients of $Q(z)$, in turn, are the factorial moments of the distribution). In other words, the Hadamard product of the functions $C_{\alpha}(-z)$ and $Q(z)$ evaluated at $z=1$ is equal to $\mathbb{E}[\log \Gamma(X+\alpha)]-\log \Gamma(\alpha)$.

We demonstrate another characterization of the generating function $C_{\alpha}(z)$ in Section 7 . Namely, we will show in (43) that $C_{\alpha}(-z)$ is the log-gamma expectation of a geometric distribution over $0,1, \ldots$ with mean $z$. Furthermore, in Appendix A, we present an intriguing connection between the logarithmic difference coefficients (and their generating function) and the Riemann zeta function and its generalized form, the Hurwitz zeta function. This leads to a formula for a weighted summation of the coefficients $c_{\alpha}(j)$ in terms of the digamma function and Harmonic numbers.

\section{Proof of Theorem 1}

We consider the Newton series expansion of the function $\log \Gamma(x)$ around point $\alpha$ given by (26), or equivalently, the series expansion of $f(x):=\log \Gamma(x+\alpha)$ around zero. The discrete derivative of $f$ is given by

$$
\Delta[f](x)=f(x+1)-f(x)=\log \frac{\Gamma(x+\alpha+1)}{\Gamma(x+\alpha)}=\log (x+\alpha) .
$$

Therefore, for $j>0$, the $j$ th forward difference of $f$ is the $(j-1)$ st forward difference of the $\operatorname{logarithmic}$ function $g(x):=\log (x+\alpha)$. This can be written down, via $(25)$, as

$$
\begin{aligned}
\Delta^{j}[f](0) & =\Delta^{j-1}[g](0) \\
& =-\sum_{k=0}^{j-1}\left(\begin{array}{c}
j-1 \\
k
\end{array}\right)(-1)^{j-k} \log (k+\alpha) \\
& \stackrel{(8)}{=}(-1)^{j} c_{\alpha}(j) .
\end{aligned}
$$

Recall that the factorial moment generating function of a distribution with moment generating function $M(t)$ is given by $M(\log z)$, and the coefficients of the power series expansion of this function around $z=1$ determine the factorial moments. Therefore, the power series expansion of the function $Q(z)=M(\log (z+1)$ ) (which is also understood as generating the inverse Stirling transform of the moment sequence) around $z=0$, namely $Q(z)=\sum_{j=0}^{\infty} q(j) z^{j}$, determines the factorial moments of the distribution. The $j$ th factorial moment of $X$ sampled from the distribution defined by $M$ is given by $\mathbb{E}\left[(X)_{j}\right]=j ! q(j)$, where $(X)_{j}$ denotes falling factorial. Note that $q(0)=1$ and $q(1)=\mathbb{E}[X]=\mu$. Using (34), we can write down the (convergent) Newton expansion of $f(x)$ and take its expectation using the information $Q(z)$ gives on the factorial moments as follows:

$$
\begin{aligned}
\mathbb{E} \log \Gamma(X+\alpha) & \stackrel{(26)}{=} \mathbb{E}\left[\log \Gamma(\alpha)+\sum_{j=1}^{\infty}\left(\begin{array}{c}
X \\
j
\end{array}\right)(-1)^{j} c_{\alpha}(j)\right] \\
& =\log \Gamma(\alpha)+\sum_{j=1}^{\infty}(-1)^{j} q(j) c_{\alpha}(j) .
\end{aligned}
$$


This proves (3). In order to derive (4), we use (27) in the above result for $j>1$, and noting that $c_{\alpha}(1)=-\log \alpha$, which gives

$$
\mathbb{E} \log \Gamma(X+\alpha)=\log \Gamma(\alpha)+\mu \log (\alpha)+\sum_{j=2}^{\infty}(-1)^{j} q(j) \int_{0}^{\infty} \frac{\left(1-e^{-t}\right)^{j-1} e^{-\alpha t}}{t} d t
$$

Recall that $M(-t)=Q\left(e^{-t}-1\right)$, and using this in the above expression, we may change the order of (convergent) summation and integration and write

$$
\begin{aligned}
\mathbb{E}[\log \Gamma(X+\alpha)] & =\log \Gamma(\alpha)+\mu \log \alpha+\int_{0}^{\infty} \frac{e^{-\alpha t}}{t\left(1-e^{-t}\right)} \sum_{j=2}^{\infty}(-1)^{j} q(j)\left(1-e^{-t}\right)^{j} d t \\
& =\log \Gamma(\alpha)+\mu \log \alpha+\int_{0}^{\infty} \frac{e^{-\alpha t}}{t\left(1-e^{-t}\right)}\left(Q\left(e^{-t}-1\right)-1-\mu\left(e^{-t}-1\right)\right) d t \\
& \stackrel{(28)}{=} \log \Gamma(\alpha)+\int_{0}^{\infty}\left(\frac{\mu e^{-t}-\mu e^{-\alpha t}}{t}+\frac{e^{-\alpha t}(M(-t)-1)}{t\left(1-e^{-t}\right)}+\frac{\mu e^{-\alpha t}}{t}\right) d t \\
& =\log \Gamma(\alpha)+\int_{0}^{\infty}\left(\frac{\mu e^{-t}}{t}-\frac{e^{-\alpha t}(1-M(-t))}{t\left(1-e^{-t}\right)}\right) d t,
\end{aligned}
$$

which proves (4). Now, (5) can be simply verified by rewriting the above integral expression in terms of the variable $z=e^{-t}-1$. Let $\bar{Q}(z):=Q(z)-\mu z-1=\sum_{j=2}^{\infty} q(j) z^{j}$. We have $t=-\log (1+z)$ and $d t=-d z /(1+z)=-e^{t} d z$ so that (4) becomes

$$
\begin{aligned}
\mathbb{E}[\log \Gamma(X+\alpha)] & =\log \Gamma(\alpha)+\int_{0}^{\infty}\left(\frac{\mu e^{-t}}{t}-\frac{\mu e^{-\alpha t}}{t}\right) d t-\int_{0}^{1} \frac{(1-z)^{\alpha-1}}{z \log (1-z)} \bar{Q}(-z) d z \\
& \stackrel{(28)}{=} \log \Gamma(\alpha)+\mu \log \alpha-\int_{0}^{1} \frac{(1-z)^{\alpha-1}}{z \log (1-z)} \bar{Q}(-z) d z,
\end{aligned}
$$

which proves (5). In fact, applying the same change of variables to (27) shows that, for $j>1$,

$$
c_{\alpha}(j)=-\int_{0}^{1} \frac{(1-z)^{\alpha-1} z^{j-1}}{\log (1-z)} d z
$$

In order to derive (6), we repeat the Newton expansion but directly on the logarithmic function $g$, noting, from (34), that $\Delta^{j}[g](0)=(-1)^{j+1} c_{\alpha}(j+1)$. So we have

$$
\begin{aligned}
\mathbb{E} \log (X+\alpha) & \stackrel{(26)}{=} \mathbb{E}\left[\sum_{j=0}^{\infty}\left(\begin{array}{c}
X \\
j
\end{array}\right)(-1)^{j+1} c_{\alpha}(j+1)\right] \\
& =\sum_{j=1}^{\infty}(-1)^{j} q(j-1) c_{\alpha}(j) .
\end{aligned}
$$

Finally, (7) immediately follows by writing $\log (X+\alpha)$ in integral form using (28) and taking the expectation of the integrand.

\section{Entropy of the Poisson distribution}

As the first application of Theorem 1, consider a Poisson distributed random variable $X$ with mean $\lambda$, and define $E_{\text {Poi }}(\lambda):=\mathbb{E}[\log X !]$, so that we have $H_{\text {Poi }}(\lambda):=H(X)=-\lambda \log (\lambda / e)+E_{\text {Poi }}(\lambda)$. The 
generating function of the distribution is $M(t)=\exp \left(\lambda\left(e^{t}-1\right)\right)$, so we have

$$
Q(z)=M(\log (z+1))=e^{\lambda z}=\sum_{j=0}^{\infty} \frac{\lambda^{j}}{j !} z^{j}
$$

Theorem 1, applied with $\alpha=1$ in (3), now directly implies that

$$
E_{\text {Poi }}(\lambda)=\sum_{j=2}^{\infty} \frac{c(j)}{j !}(-\lambda)^{j}
$$

where we recall the shorthand $c(j):=c_{1}(j)$, thus recovering the Maclaurin series expansion of $E_{\text {Poi }}$ in (2). Note that this expansion is the same as the Newton series expansion of the function $\log (\lambda !)$, with factorial powers of $\lambda$ replaced by actual powers. Since $(c(j))_{j=2}^{\infty}$ is a decreasing sequence, a simple ratio test reveals that the above power series expansion absolutely converges for all $\lambda>0$. Furthermore, observe that the integral expression (15) can be immediately recovered from (5) in Theorem 1.

While we do not know of a representation of the function $E_{\mathrm{Poi}}(\lambda)$ in terms of elementary or natural special functions, we may see that its Laplace transform (which we will revisit later in Section 7) takes an interesting form. Let $\hat{E}_{\mathrm{Poi}}(z)$ denote the Laplace transform of $E_{\mathrm{Poi}}(\lambda)$. By the Laplace transform formula for power series (namely, $\mathcal{L}\left\{\lambda^{j}\right\}=j !(1 / z)^{j+1}$ ), and using (35), we may write

$$
\hat{E}_{\mathrm{Poi}}(z)=-\sum_{j=2}^{\infty} c(j)(-1 / z)^{j+1} \stackrel{(33)}{=} \frac{-1}{z(1+z)} \Phi^{\prime}\left(\frac{1}{1+z}\right) .
$$

Combined with the Laplace transform of $-\lambda \log (\lambda / e)$, which is $(\gamma+\log z) / z^{2}$ (where $\gamma \approx 0.57721$ is the Euler-Mascheroni constant), we conclude the following:

Theorem 2. The Laplace transform of the entropy function $H_{\mathrm{Poi}}(\lambda)$ is given by

$$
\mathcal{L}\left\{H_{\mathrm{Poi}}\right\}(z)=\frac{\gamma+\log z}{z^{2}}-\frac{1}{z(1+z)} \Phi^{\prime}\left(\frac{1}{1+z}\right),
$$

where $\Phi$ is derivative of the polylogarithm function defined in (31).

Remark 3. An alternative way of proving Theorem 2 is to directly start from (1) and use the frequency shifting property of the Laplace transform and then the convolution property of generating functions (applied on $\Phi^{\prime}(z)$ and the geometric series $1 /(1-z)$ ) in order to generate the sequence $(\log k !)_{k=0}^{\infty}$ from $(\log (1+k))_{k=0}^{\infty}$.

\section{Entropy of the binomial distribution}

Recall that the binomial distribution with parameters $n, p$ is defined by the probability mass function in (10). In general, $n$ need not be an integer. This distribution has mean $n p$, variance $n p(1-p)$, and moment generating function $\left(1-p+p e^{t}\right)^{n}$.

Let $X$ be a random variable distributed according to a binomial distribution with parameters $n, p$. Similar to the Poisson distribution, the difficulty in computing $H(X)$ is captured by the computation of

$$
E_{\mathrm{Bin}}(n, p):=\mathbb{E}[\log X !],
$$

and, directly from (10), we can see that $H(X)=n h(p)-\log \Gamma(n+1)+E_{\mathrm{Bin}}(n, p)+E_{\mathrm{Bin}}(n, 1-p)$. 
Using (4), we can immediately write down an integral representation of $E_{\mathrm{Bin}}(n, p)$ :

$$
E_{\mathrm{Bin}}(n, p)=\int_{0}^{\infty}\left(\frac{n p e^{-t}}{t}-\frac{e^{-t}\left(1-\left(1-p+p e^{-t}\right)^{n}\right)}{t\left(1-e^{-t}\right)}\right) d t
$$

so that we have the integral representation of $H(X)$ given by $(16)$ :

$$
H(X)=n h(p)+\int_{0}^{\infty}\left(\frac{\left(1-p+p e^{-t}\right)^{n}+\left(p+(1-p) e^{-t}\right)^{n}-e^{-n t}-1}{t\left(e^{t}-1\right)}\right) d t .
$$

Now, observe that the function $Q(z)$ in Theorem 1 is $Q(z)=(1-p z)^{n}=\sum_{j=0}^{\infty}\left(\begin{array}{c}n \\ j\end{array}\right)(-p z)^{j}$. Therefore, by (3), we have

$$
E_{\mathrm{Bin}}(n, p)=\sum_{j=2}^{\infty}\left(\begin{array}{l}
n \\
j
\end{array}\right) c(j)(-p)^{j}=\sum_{j=2}^{\infty} \frac{c(j)}{j !}(-p)^{j}(n)_{j},
$$

where $(n)_{j}$ denotes the falling factorial. This gives us the following series expansion for the entropy:

$$
H(X)=n h(p)+\sum_{j=2}^{\infty}\left(\begin{array}{l}
n \\
j
\end{array}\right)(-1)^{j} c(j)\left(p^{j}+(1-p)^{j}-1\right)
$$

thus confirming (17).

Remark 4. Observe the remarkable similarity between (37) and the analogous quantity $E_{\mathrm{Poi}}$ for the Poisson distribution in (2): Indeed, (37) is obtained from (2) by letting $\lambda=n p$ and replacing powers of $n$ with factorial powers of the same order. This makes intuitive sense as the Poisson distribution is the limiting case for the binomial distribution as $n$ tends to infinity and the expectation $n p$ is fixed to the desired parameter $\lambda$, in which case falling factorials $(n)_{j}$ are within a multiplicative factor $1+O(1 / n)$ of the corresponding actual powers $n^{j}$, a factor that tends to 1 as $n$ grows.

\section{Entropy of the beta-binomial distribution}

A beta-binomial distribution is defined by positive parameters $n, \alpha, \beta$ (where $n$ is typically an integer) and probability mass function $\operatorname{BBin}(k ; n, \alpha, \beta)$ in (11). The moment generating function for this distribution is

$$
M(t)={ }_{2} F_{1}\left(-n, \alpha ; \alpha+\beta ; 1-e^{t}\right),
$$

where $t<\log 2$ and ${ }_{2} F_{1}$ denotes the hypergeometric function defined as

$$
{ }_{2} F_{1}(a, b ; c ; z)=\sum_{j=0}^{\infty} \frac{a^{(j)} b^{(j)}}{c^{(j)}} \frac{z^{j}}{j !}
$$

and $a^{(j)}$ is the rising factorial. Let $X$ be a random variable with beta distribution defined by parameters $n, \alpha, \beta$. The distribution of $X$ is a compound distribution generated by sampling a random parameter $p \in(0,1)$ according to a beta distribution with parameters $\alpha, \beta$, and subsequently, drawing from a binomial distribution defined by parameters $n, p$. The expectation of $p$ is $\alpha /(\alpha+\beta)$ (and thus $\mathbb{E}[X]=n \alpha /(\alpha+\beta)$ ). For a fixed ratio $\alpha /(\alpha+\beta)$, the variance of $p$ decreases as $\alpha$ grows, and thus the binomial distribution is a limiting case of the beta-binomial distribution when $\alpha \rightarrow \infty$ and $\alpha /(\alpha+\beta)=p$. 
Similar to the Poisson and binomial distributions, the difficulty in computing the entropy of a beta-binomial distribution lies at the computation of

$$
E_{\mathrm{BBin}}(n, \alpha, \beta, r):=\mathbb{E}[\log \Gamma(X+r)],
$$

for a fixed parameter $r$ (which is either $1, \alpha$, or $\beta$ ). Using this notation, and noting that the distribution of $n-X$ is given by $\operatorname{BBin}(k ; n, \beta, \alpha)$, the entropy can be written as

$$
\begin{aligned}
H(X) & =\log B(\alpha, \beta)+\mathbb{E}\left[\log \frac{\Gamma(n+\alpha+\beta) \Gamma(X+1) \Gamma(n-X+1)}{\Gamma(n+1) \Gamma(X+\alpha) \Gamma(n-X+\beta)}\right] \\
& =\log B(\alpha, \beta)+\log \frac{\Gamma(n+\alpha+\beta)}{\Gamma(n+1)}+E_{\mathrm{BBin}}(n, \alpha, \beta, 1)+E_{\mathrm{BBin}}(n, \beta, \alpha, 1) \\
& -E_{\mathrm{BBin}}(n, \alpha, \beta, \alpha)-E_{\mathrm{BBin}}(n, \beta, \alpha, \beta) .
\end{aligned}
$$

As before, we may use (4) and (5), noting that $Q(z)={ }_{2} F_{1}(-n, \alpha ; \alpha+\beta ; z)$, to derive an integral representation of the function $E_{\mathrm{BBin}}$ as

$$
\begin{aligned}
E_{\mathrm{BBin}}(n, \alpha, \beta, r) & \stackrel{(4)}{=} \log \Gamma(r)+\int_{0}^{\infty}\left(\frac{n \alpha e^{-t}}{t(\alpha+\beta)}-\frac{e^{-r t}\left(1-{ }_{2} F_{1}\left(-n, \alpha ; \alpha+\beta ; 1-e^{t}\right)\right)}{t\left(1-e^{-t}\right)}\right) d t \\
& \stackrel{(5)}{=} \log \Gamma(r)+\frac{n \alpha \log r}{\alpha+\beta}+\int_{0}^{1} \frac{(1-z)^{r-1}\left({ }_{2} F_{1}(-n, \alpha ; \alpha+\beta ;-z)+n \alpha /(\alpha+\beta)-1\right)}{z \log (1-z)} d z,
\end{aligned}
$$

which confirms (19). By (38), the coefficient of $z^{j}$ in the series expansion of $Q(z)$ is equal to

$$
\frac{(-1)^{j} \alpha^{(j)}}{(\alpha+\beta)^{(j)}}\left(\begin{array}{l}
n \\
j
\end{array}\right)
$$

Therefore, using (3), we obtain the series expansion of $E_{\mathrm{BBin}}(n, \alpha, \beta, r)$ in $(21)$ :

$$
E_{\mathrm{BBin}}(n, \alpha, \beta, r)=\log \Gamma(r)+\sum_{j=2}^{\infty}(-1)^{j} c_{r}(j) \frac{\alpha^{(j)}}{(\alpha+\beta)^{(j)}}\left(\begin{array}{c}
n \\
j
\end{array}\right) .
$$

Observe that, letting $p:=\alpha /(\alpha+\beta)$, if $p$ is fixed while $\alpha$ grows large, the ratio $\alpha^{(j)} /(\alpha+\beta)^{(j)}$ becomes $p^{j}(1+O(1 / \alpha))$, and thus, the terms in (40) converge to those in (37) (albeit (37) is written for the special case $r=1$ ). This is consistent with the fact that the binomial distribution is the limiting distribution of the beta-binomial distribution for fixed $p$ as $\alpha$ tends to infinity. Plugging this result into (39), we derive a series expansion for the entropy of beta-binomial distribution:

$$
\begin{aligned}
H(X) & =\log \left(\frac{\Gamma(\alpha) \Gamma(\beta)}{\Gamma(\alpha+\beta)} \frac{\Gamma(n+\alpha+\beta)}{n \Gamma(n)}\right)-\log \Gamma(\alpha)-\log \Gamma(\beta) \\
& +\sum_{j=2}^{\infty}\left(\begin{array}{c}
n \\
j
\end{array}\right) \frac{(-1)^{j}}{(\alpha+\beta)^{(j)}}\left(\left(c(j)-c_{\alpha}(j)\right) \alpha^{(j)}+\left(c(j)-c_{\beta}(j)\right) \beta^{(j)}\right) \\
& =-\log (n B(n, \alpha+\beta))+\sum_{j=2}^{\infty}\left(\begin{array}{c}
n \\
j
\end{array}\right) \frac{(-1)^{j}}{(\alpha+\beta)^{(j)}}\left(\left(c(j)-c_{\alpha}(j)\right) \alpha^{(j)}+\left(c(j)-c_{\beta}(j)\right) \beta^{(j)}\right),
\end{aligned}
$$

which proves (18). 


\section{Entropy of the negative binomial distribution}

Recall that the negative binomial distribution is defined by the probability mass function NBin $(k ; r, p)$ in (12), for parameters $r>0$ and $p \in(0,1)$, and has mean $p r /(1-p)$. When $r$ is an integer, the distribution captures an independent summation of $r$ identical, geometrically distributed, random variables. The moment generating function of the distribution is given by

$$
M(t)=\left(\frac{1-p}{1-p e^{t}}\right)^{r}
$$

Let $X$ be a negative binomial random variable with parameters $r$ and $p$, and define $E_{\mathrm{Bin}}(r, p, \alpha):=$ $\mathbb{E}[\log \Gamma(X+\alpha)]$. Using this notation, we may write

$$
\begin{aligned}
H(X) & =-r \log (1-p)-p r(\log p) /(1-p)+\log \Gamma(r)+E_{\mathrm{Bin}}(r, p, 1)-E_{\mathrm{Bin}}(r, p, r) \\
& =\frac{r h(p)}{1-p}+\log \Gamma(r)+E_{\mathrm{Bin}}(r, p, 1)-E_{\mathrm{Bin}}(r, p, r) .
\end{aligned}
$$

Let $q:=p /(1-p)$. We now consider the function $Q(z)=M(\log (z+1))$, which can be written as

$$
\begin{aligned}
Q(z) & =\left(\frac{1-p}{1-p(z+1)}\right)^{r}=\left(\frac{1}{1-q z}\right)^{r} \\
& =\sum_{j=0}^{\infty}\left(\begin{array}{c}
-r \\
j
\end{array}\right)(-q z)^{j}=\sum_{j=0}^{\infty}\left(\begin{array}{c}
j+r-1 \\
j
\end{array}\right)(q z)^{j},
\end{aligned}
$$

and thus the $j$ th power series coefficient of $Q(z)$; i.e., the $j$ th factorial moment of the distribution is equal to

$$
j !\left(\begin{array}{c}
j+r-1 \\
j
\end{array}\right) q^{j}
$$

We are now ready to apply Theorem 1 and conclude, using (3), that

$$
E_{\mathrm{NBin}}(r, p, \alpha)=\log \Gamma(\alpha)+\sum_{j=1}^{\infty}\left(\begin{array}{c}
j+r-1 \\
j
\end{array}\right)(-q)^{j} c_{\alpha}(j) .
$$

Plugging this result into (41), we thus have verified (22):

$$
H(X)=\frac{r h(p)}{1-p}+\sum_{j=1}^{\infty}\left(\begin{array}{c}
j+r-1 \\
j
\end{array}\right)\left(\frac{p}{p-1}\right)^{j}\left(c(j)-c_{r}(j)\right) .
$$

Notice that, when $r=1$, the above expression reduces to $H(X)=h(p) /(1-p)$, which is the entropy of a geometric distribution.

Let us now consider the logarithmic expectation of the distribution. Define $E_{\mathrm{NBin}}^{\prime}(r, p, \alpha):=$ $\mathbb{E}[\log (X+\alpha)]$. Similar to $E_{\mathrm{NBin}}$, we can use $(6)$ to expand $E_{\mathrm{NBin}}^{\prime}$ as

$$
\begin{aligned}
E_{\mathrm{NBin}}^{\prime}(r, p, \alpha) & =-\sum_{j=1}^{\infty}\left(\begin{array}{c}
j+r-2 \\
j-1
\end{array}\right)(-q)^{j-1} c_{\alpha}(j) \\
& =\frac{1}{q} \sum_{j=0}^{\infty}\left(\begin{array}{c}
j+r-2 \\
j-1
\end{array}\right)(-q)^{j} c_{\alpha}(j) .
\end{aligned}
$$


When $r=1$, this reduces to the logarithmic expectation of a geometric distribution. Let us write

$$
\begin{aligned}
E_{\mathrm{Geom}}(p, \alpha) & :=E_{\mathrm{Bin}}^{\prime}(1, p, \alpha) \\
& \stackrel{(44)}{=} \frac{1}{q} \sum_{j=0}^{\infty}(-q)^{j} c_{\alpha}(j) \\
& \stackrel{(32)}{=} \frac{1}{q} C_{\alpha}(-q) \\
& \stackrel{(33)}{=}-\frac{1}{1+q} \Phi_{\alpha}^{\prime}\left(\frac{q}{1+q}\right) \\
& =(p-1) \Phi_{\alpha}^{\prime}(p),
\end{aligned}
$$

where $C_{\alpha}(\cdot)$ is the generating function of the logarithmic difference coefficients defined in (32) and $\Phi_{\alpha}^{\prime}$ is the derivative of the Lerch transcendent (and the polylogarithm for $\alpha=1$ ) defined in (30) and (31). We have thus shown the following characterization of the generating function of the logarithmic difference coefficients:

Corollary 5. Let $E_{\mathrm{Geom}}(p, \alpha):=\mathbb{E}[\log (X+\alpha)]$ where $X \geq 0$ is geometrically distributed with mean $q=p /(1-p)$. Then,

$$
q E_{\mathrm{Geom}}(p, \alpha)=C_{\alpha}(-q),
$$

where $C_{\alpha}(\cdot)$ is the generating function of the coefficients $c_{\alpha}(j)$ defined in (8).

By combining Corollary 5, (33), and (36), and simple manipulations, we arrive at the following curious result:

Theorem 6. Let $E_{\mathrm{Geom}}(p):=\mathbb{E}[\log (X+1)]$, where $X \geq 0$ is geometrically distributed with mean $p /(1-p)$. Similarly, define $E_{\mathrm{Poi}}(\lambda)=\mathbb{E}[\log Y !]$, where $Y$ is a Poisson-distributed random variable with mean $\lambda$. Then, the Laplace transform of $E_{\mathrm{Poi}}$ is given by

$$
\mathcal{L}\left\{E_{\mathrm{Poi}}\right\}(z)=\frac{1}{z^{2}} E_{\mathrm{Geom}}\left(\frac{1}{1+z}\right) .
$$

In order to derive the integral representation for $H(X)$ given in (23), we may apply Theorem 1 (specifically, (5)) with the choice of $Q(z)$ in (42) to write, recalling that $\mathbb{E}[X]=q r=r p /(1-p)$,

$$
E_{\mathrm{NBin}}(r, p, \alpha)=\log \Gamma(\alpha)+q r \log \alpha-\int_{0}^{1} \frac{(1-z)^{\alpha-1}}{z \log (1-z)}\left((1+q z)^{-r}+q r z-1\right) d z,
$$

and thus, plugging the above into (41),

$$
\begin{aligned}
H(X) & =\frac{r h(p)}{1-p}+\log \Gamma(r)-\int_{0}^{1} \frac{(1+q z)^{-r}+q r z-1}{z \log (1-z)} d z \\
& -\log \Gamma(r)-q r \log r+\int_{0}^{1} \frac{(1-z)^{r-1}\left((1+q z)^{-r}+q r z-1\right)}{z \log (1-z)} d z \\
& =\frac{r(h(p)-p \log r)}{1-p}+\int_{0}^{1} \frac{\left((1-z)^{r-1}-1\right)\left((1+p z /(1-p))^{-r}+p r z /(1-p)-1\right)}{z \log (1-z)} d z .
\end{aligned}
$$

which recovers $(23)$. 


\section{Entropy of the hypergeometric distribution}

The hypergeometric distribution is regarded as an analogue of the binomial distribution, with the difference that the Bernoulli trials are performed without replacement. Namely, the parameters $n, N, K$ are respectively regarded as the number of trials, population size, and number of success states in the population. For each trial, an item is drawn, uniformly at random, from the population, in which initially $K$ specific items are marked as "success states". The trial results in a success if a success state is drawn, and is otherwise a failure. After each trial, the drawn item is discarded from the population, and the resulting random variable counts the number of successful trials. The probability mass function for this distribution is given in (13). The distribution contains the binomial distribution (and thus, the Poisson distribution) as a limiting case when the ratio $K / N=p$ is a given success probability and $N$ tends to infinity. While the parameters $N, K, n$ are normally set to be integers, the distribution (over $k=0,1, \ldots$ ) still normalizes to a total mass of 1 and is thus well defined even if the parameters may be chosen to be non-integral, in which case the binomial coefficients should be understood in terms of the beta function.

As in the beta-binomial distribution, the moment generating function for the hypergeometric distribution involves the hypergeometric function, and is given by the expression

$$
M(t)=\left(\begin{array}{c}
N-K \\
n
\end{array}\right){ }_{2} F_{1}\left(-n,-K ; N-K-n+1 ; e^{t}\right) /\left(\begin{array}{c}
N \\
n
\end{array}\right) .
$$

Thus in this case, the function $Q(z)=M(\log (z+1))$ is equal to

$$
Q(z)=1+\sum_{j=1}^{\infty} q(j) z^{j}=\left(\begin{array}{c}
N-K \\
n
\end{array}\right){ }_{2} F_{1}(-n,-K ; N-K-n+1 ; 1+z) /\left(\begin{array}{l}
N \\
n
\end{array}\right) .
$$

Despite the seemingly complicated expression, the coefficients $q(j)$, which are the factorial moments of the distributions, we known to have a simple form [Pot53]:

$$
q(j)=\frac{(K)_{j}(n)_{j}}{(N)_{j}}
$$

where $(a)_{b}=\left(\begin{array}{l}a \\ b\end{array}\right) b$ ! denotes the falling factorial. We are now ready to apply Theorem 1 to show that, letting $X$ be drawn from the hypergeometric distribution with probability mass function (13),

$$
E_{N, K, n}:=\mathbb{E}[\log X !]=\sum_{j=2}^{\infty}(-1)^{j} q(j) c(j)=\sum_{j=2}^{\infty}(-1)^{j} c(j) \frac{(K)_{j}(n)_{j}}{(N)_{j}} .
$$

The entropy of $X_{N, K, n}$ can be directly expressed from (13) as

$$
\begin{aligned}
H(X)=\log \left(\begin{array}{l}
N \\
n
\end{array}\right)-\log & K !-\log (N-K) ! \\
& +\mathbb{E}[\log X !+\log (n-X) !+\log (K-X) !+\log (N-K-n+X) !] .
\end{aligned}
$$

We now used the following basic symmetries in the distribution:

$\mathrm{HG}(k ; N, K, n)=\mathrm{HG}(n-k ; N, N-K, n)=\mathrm{HG}(K-k ; N, K, N-n)=\mathrm{HG}(N-K-n+k ; N, N-K, N-n)$,

where the last identity is obtained by combining the first two. This, in turn, implies that

$$
\begin{aligned}
\mathbb{E}[\log (n-X) !] & =E_{N, N-K, n} \\
\mathbb{E}[\log (K-X) !] & =E_{N, K, N-n} \\
\mathbb{E}[\log (N-K-n+X) !] & =E_{N, N-K, N-n} .
\end{aligned}
$$


Plugging this result back in (48), combined with (47), leads to the following series for the entropy of the hypergeometric distribution,

$$
\begin{aligned}
H(X) & =\log \left(\begin{array}{c}
N \\
n
\end{array}\right)-\log K !-\log (N-K) ! \\
& +\sum_{j=2}^{\infty} \frac{(-1)^{j} c(j)}{(N)_{j}}\left((K)_{j}(n)_{j}+(N-K)_{j}(n)_{j}+(K)_{j}(N-n)_{j}+(N-K)_{j}(N-n)_{j}\right),
\end{aligned}
$$

proving (24).

\section{Discussion}

In this work, we studied a general method for deriving series expansions and integral representations for logarithmic and log-gamma expectations of arbitrary distributions. As a result, we obtained entropy expressions for several fundamental distributions, including the Poisson, binomial, betabinomial, and negative binomial distributions. It is natural to ask whether the technique can be extended to derive clean expressions for the entropy of other distributions. Another natural direction is whether the techniques can be used to obtain clean, general, and high-precision estimates of the entropy functions in terms of elementary functions.

We have also discovered connections between logarithmic expectations of different distributions, and moreover, connections between them and generalizations of the Riemann zeta function via the Laplace transform. An intriguing question is whether such curious connections with functional transforms are isolated facts or can be further developed into a richer theory. Finally, our work calls for a further study and better understanding of the logarithmic difference coefficients $c_{\alpha}(j)$, which are also of interest in the analytic study of zeta functions.

\section{Acknowledgement}

The author thanks an anonymous reviewer for comments on related works [Kne98, JS99, CGKK13].

\section{References}

[AW12] R. Atar and T. Weissman, Mutual information, relative entropy, and estimation in the Poisson channel, IEEE Transactions on Information Theory 58 (2012), no. 3, 1302-1318.

[CGKK13] J. Cichoń, Z. Gołębiewski, M. Kardas, and M. Klonowski, On delta-method of moments and probabilistic sums, Proceedings of the tenth workshop on analytic algorithmics and combinatorics (ANALCO), 2013, pp. 91-98.

[Che18] M. Cheraghchi, Capacity upper bounds for deletion-type channels, Proceedings of the 50th annual ACM SIGACT Symposium on Theory of Computing (STOC), 2018, pp. 493-506.

[CR18a] M. Cheraghchi and J. Ribeiro, Improved capacity upper bounds for the discrete-time Poisson channel, Proceedings of the IEEE International Symposium on Information Theory, 2018.

[CR18b] _ Sharp analytical capacity upper bounds for sticky and related channels, CoRR abs/1806.06218 (2018).

[CT06] T. M. Cover and J. A. Thomas, Elements of information theory, Second, John Wiley and Sons, 2006.

[DS12] M. H. DeGroot and M. J. Schervish, Probability and statistics, Fourth, Addison-Wesley, 2012.

[DV98] A. G. D'yachkov and P. A. Vilenkin, Asymptotics of the Shannon and Renyi entropies for sums of independent random variables, Proceedings of IEEE International Symposium on Information Theory, 1998, pp. 376 . 
[EB88] R. J. Evans and J. Boersma, The entropy of a Poisson distribution (C. Robert Appledorn), SIAM Review 30 (1988), no. 2, 314-317.

[EMOT53] A. Erdélyi, W. Magnus, F. Oberhettinger, and F. G. Tricomi, Higher transcendental functions, Vol. 1, McGraw-Hill, 1953.

[Has30] H. Hasse, Ein Summierungsverfahren für die Riemannsche $\zeta$-Reihe, Mathematische Zeitschrift 32 (1930), no. 1, 458-464.

[JS99] P. Jacquet and W. Szpankowski, Entropy computations via analytic depoissonization, IEEE Transactions on Information Theory 45 (1999), no. 4, 1072-1081.

[Kne98] C. Knessl, Integral representations and asymptotic expansions for Shannon and Renyi entropies, Applied Mathematics Letters 11 (1998), no. 2, 69-74.

[Knu73] D. Knuth, The art of computer programming, Vol. 3, Addison-Wesley, 1973.

[Mar07] A. Martinez, Spectral efficiency of optical direct detection, JOSA B 24 (2007), no. 4, 739-749.

[Mit08] M. Mitzenmacher, A survey of results for deletion channels and related synchronization channels, Proceedings of SWAT 2008: 11th scandinavian workshop on algorithm theory, 2008, pp. 1-3.

[Pot53] R. B. Potts, Note on the factorial moments of standard distributions, Australian Journal of Physics 6 (1953), 498.

[Sha90] S. Shamai, Capacity of a pulse amplitude modulated direct detection photon channel, IEE Proceedings I Communications, Speech and Vision 137 (1990), no. 6, 424-430.

[Ver08] S. Verdú, Shannon and Poisson, Symposium on foundations of wireless networks and beyond, 2008.

[Ver99] _ Poisson communication theory, The international Technion Communication Day in honor of Israel Bar-David, 1999. Invited talk.

\section{A Connection between the $c_{\alpha}(j)$, the Riemann zeta function, and Harmonic numbers}

In this appendix, we observe a connection between the logarithmic difference coefficients and the Riemann zeta function, leading to an interesting formula for a harmonically weighted summation of the coefficients.

The following convergent Newton expansion series for Hurwitz zeta function $\zeta(s, \alpha):=\sum_{n=0}^{\infty}(n+$ $\alpha)^{-s}$ (which reduces to the Riemann zeta function $\zeta(s)$ at $\alpha=1$ ) was given by Hasse [Has30]: For all $\alpha>0$ and $s \neq 1$,

$$
\zeta(s, \alpha)(s-1)=\sum_{j=1}^{\infty} \frac{1}{j} \sum_{k=0}^{j-1}\left(\begin{array}{c}
j-1 \\
k
\end{array}\right)(-1)^{k}(k+\alpha)^{1-s} .
$$

Taking the derivative of the above in $s$, denoting $\zeta^{\prime}(s, \alpha):=\frac{d}{d s} \zeta(s, \alpha)$, gives

$$
\zeta(s, \alpha)+(s-1) \zeta^{\prime}(s, \alpha)=-\sum_{j=1}^{\infty} \frac{1}{j} \sum_{k=0}^{j-1}\left(\begin{array}{c}
j-1 \\
k
\end{array}\right)(-1)^{k}(k+\alpha)^{1-s} \log (k+\alpha) .
$$

Observe, from (8), that $c_{\alpha}(j)$ is the inner summation on the right hand side at $s=1$. The limit of the left hand side of the above equality at $s=1$ can be deduced from the first two terms of the Laurent series expansion of the Hurwitz zeta function,

$$
\zeta(s, \alpha)=\frac{1}{s-1}-\psi(\alpha)+\sum_{n=1}^{\infty} a_{n}(s-1)^{n}
$$

and is thus equal to $-\psi(\alpha)$, where $\psi(\alpha)=\frac{d}{d \alpha} \log \Gamma(\alpha)=H_{\alpha-1}-\gamma$ is the digamma function, $\gamma \approx 0.57721$ is the Euler-Mascheroni constant, and $H_{k}$ is the $k$ th Harmonic number. When $\alpha$ is 
a positive integer, we thus have that the left identity at $s \rightarrow 1$ is equal to $-\gamma+\sum_{i=1}^{\alpha-1} \frac{1}{i}$ (and $-\gamma$ when $\alpha=1$ ). We conclude the following identity on the logarithmic difference coefficients: for all $\alpha>0$,

$$
\sum_{j=1}^{\infty} c_{\alpha}(j) / j=\psi(\alpha)=\gamma-H_{\alpha-1}
$$

Note that the left hand side can be rewritten as $\left.\int_{0}^{t} \frac{C_{\alpha}(z)}{z} d z\right|_{t=1}$, where $C_{\alpha}(z)$ is the generating function of the $c_{\alpha}(j)$ defined in $(32)$. 